\title{
IMPACTO DA OFERTA DE POLEIROS SOBRE O BEM-ESTAR DE FRANGOS DE CORTE
}

\author{
(Impact of Perch Usage on Broiler Welfare) \\ NORDI, W.M. ${ }^{1,2}$; YAMASHIRO, K. ${ }^{1,2}$; KLANK, M. ${ }^{1,2}$; CARDOZO, E.C. ${ }^{3}$; DAHLKE, F. ${ }^{4}$; \\ DITTRICH, R.L. ${ }^{5}$ MOLENTO, C.F.M. ${ }^{1,6}$ \\ 'LABEA - Laboratório de Bem-Estar Animal, SCA, UFPR; \\ ${ }^{2}$ Acadêmicas do Curso de Zootecnia, UFPR; \\ ${ }^{3}$ Mestrando, Programa de Pós-Graduação em Ciências Veterinárias, SCA, UFPR; \\ ${ }^{4}$ Professor, Departamento de Zootecnia, SCA, UFPR; \\ ${ }^{5}$ Professora, Departamento de Medicina Veterinária, SCA, UFPR; \\ ${ }^{6}$ Coordenadora do LABEA, Professora, Departamento de Zootecnia, SCA, UFPR, Rua dos Funcionários, \\ 1540, Curitiba, Paraná, CEP 80035-050, carlamolento@yahoo.com, 4133505788.
}

\begin{abstract}
RESUMO - A produção industrial de frangos de corte desafia de maneira importante o bem-estar das aves. O objetivo deste foi melhorar o grau de bem-estar de frangos de corte, através do fornecimento de poleiros e da avaliação de seu impacto no bem-estar dos animais. Foram utilizados 360 frangos de corte, machos, criados de 1 a 42 dias de idade, em dois tratamentos com seis repetições de 30 aves cada, sendo $T 1$ sem poleiros e T2 com três poleiros por box. Foram realizadas observações das atividades comportamentais por registro instantâneo do tipo varredura. Exames físicos para condição das penas do peito e distúrbios nas pernas foram realizados aos 40 dias de idade. Foram avaliados: consumo de ração, ganho de peso, peso ao abate e conversão alimentar. Foi observada freqüência de empoleiramento crescente com a idade das aves, de $0,3 \%, 2,6 \%$ e $5,1 \%$ na quarta, quinta e sexta semanas de idade, respectivamente. Durante as duas últimas semanas de vida, as ocorrências de ciscar foram mais freqüentes e os eventos de comportamento agonista foram menos freqüentes no tratamento com poleiros $(P<0,05)$. Os resultados não foram conclusivos quanto ao efeito dos tratamentos na condição das penas do peito e distúrbios de pernas. Em geral, não houve diferença nos parâmetros zootécnicos $(P>0,05)$. Conclui-se que, nas condições deste experimento, a oferta de poleiros resulta em melhoria da Liberdade Comportamental no terço final de vida das aves. O efeito dos poleiros sobre a Liberdade Sanitária deve ser estudado mais detalhadamente. Os resultados sugerem melhoria do grau de bem-estar de frangos de corte devido à oferta de poleiros.
\end{abstract}

Palavras-chave: aves; comportamento; Cinco Liberdades.

\footnotetext{
ABSTRACT - Industrial broiler production presents important challenges to the welfare of the birds. The objective of this work was to increase broiler welfare through the inclusion of perches and the evaluation of their impact on the welfare of the animals. Three hundred and sixty male birds were raised from day 1 to 42 in two
}

treatments, with six boxes per treatment and 30 birds per box, being $\mathrm{T} 1$ - boxes with no perch and T2 - boxes with three perches each. Behavioural observations were organized as instantaneous scan sampling. Forty dayold birds were subjected to physical clinical exam to register chest feather condition and leg health. Ration consumption, weight gain, slaughter weight and feed conversion were monitored. The frequency of perching events was higher as birds grew older, being $0.3 \%, 2.6 \%$ e $5.1 \%$ for the fourth, fifth and sixth weeks, respectively. During the last two weeks of life, frequency of floor pecking events was higher and of agonistic interactions was lower for broilers maintained with perches $(P<0.05)$. Results for the effect of treatments on chest feather conditions and on leg problems were inconclusive. In general, there was no treatment effect on production indexes $(P>0.05)$. In the experimental conditions, we conclude that the presence of perches results in improvements of the Behavioural Freedom for the last third of the bird's life. More research is needed regarding the perch effects on broiler's Sanitary Freedom. Results suggest improvement of broiler welfare due to the presence of perches.

Key-words: poultry; behaviour; Five Freedoms.

\section{Introdução}

A pecuária pode ser uma das atividades de maior impacto na qualidade de vida animal, especialmente se for considerado um diagnóstico de bem-estar que inclua intensidade e duração do impacto negativo sobre o bem-estar dos animais associadas ao número de indivíduos atingidos (BROOM e MOLENTO, 2004). Atualmente, a produção de frangos de muitos países é realizada de forma industrial com alta densidade populacional. A relação entre produtividade e bem-estar animal em tal sistema encontra-se deslocada fortemente à direita na curva de Mclnerney (MOLENTO, 2005). Este fato caracteriza ganho de produtividade com decréscimo significativo de qualidade de vida para os animais, sendo que as restrições importantes ao bem- 
estar das aves são: altas densidades de lotação, limitação severa de espaço, redução severa do repertório comportamental possível e alta incidência de distúrbios nas pernas e problemas de peito (FRASER e BROOM, 2002; JENSEN, 2002). Adicionalmente, existem os efeitos negativos sobre o bem-estar das aves oriundos da seleção genética para crescimento rápido, tais como as desordens metabólicas, que podem levar à ascite e morte súbita (BESSEI, 2006). Desta forma, o impacto da produção industrial na qualidade de vida das aves é severo. Em termos de número de indivíduos envolvidos, somente no Brasil, a cadeia produtiva de 2005 envolveu cerca de 4,5 bilhões de frangos (APINCO, 2006). Conseqüentemente, a produção de frangos passa a ser alvo de tentativas de melhoria no grau de bem-estar (SHEPPARD e EDGE, 2005).

Nos países da Comunidade Européia, a preocupação com o grau de bem-estar dos animais levou à existência de moratórias para a eliminação dos sistemas intensivos mais agressivos aos animais (STATUTORY INSTRUMENT, 2000). A legislação brasileira, de forma coerente com o contexto do mundo ocidental, demonstra resposta clara à demanda atual da sociedade, como pode ser constatado nas leis de estados tais como o Código de Proteção aos Animais do Paraná (LEI 14.037, 2003) e o Código Estadual de Proteção aos Animais de São Paulo (LEl 11.977, 2005), os quais incluem obrigações significativas em relação ao bem-estar de animais de produção.

A inserção de itens de enriquecimento ambiental pode ser um fator de aumento no grau de bem-estar animal (MITCHELL e KETTLEWELL, 2003). A utilização de poleiros pode ser uma alternativa viável para o enriquecimento ambiental de granjas comerciais. Os poleiros promovem uma utilização da área vertical do galpão, tendo o poder de decréscimo do impacto das altas densidades de lotação utilizadas pela indústria (NEWBERRY, 1995). Adicionalmente, os poleiros podem servir como encorajadores da execução de exercícios pelas aves (ESTEVEZ et al., 2002), oportunizando algum controle sobre ambiente, através de comportamentos naturais da espécie (NEWBERRY, 1995) e criando potencial redução de problemas físicos relacionados à falta de movimentação, tais como problemas no peito e nas pernas. Entretanto, um fator limitante para o uso de poleiros é sua aparente baixa utilização por frangos de crescimento rápido (BESSEY, 2006).

Este trabalho teve como objetivo melhorar o grau de bem-estar de frangos de corte, através do fornecimento de poleiros com motivadores.

\section{Material e Métodos}

A avaliação do grau de bem-estar das aves baseouse no conceito das Cinco Liberdades do bem-estar animal (MOLENTO, 2003; MOLENTO, 2006). Dentre estas, trabalhou-se com as Liberdades Comportamental e Sanitária.
Animais e delineamento experimental: Foram utilizados 360 frangos de corte, machos, da linhagem comercial Ross, criados do primeiro aos 42 dias de idade. As aves foram alojadas em 12 boxes de $4,20 \mathrm{~m}^{2}$ (1,50 $\mathrm{m} \times 2,80 \mathrm{~m})$, em densidade de 7,1 aves $/ \mathrm{m}^{2}$. Os boxes foram revestidos por cama de maravalha de 12 $\mathrm{cm}$. Ração e água foram fornecidas ad libitum, sendo que a mesma ração foi oferecida a todas as aves. $\mathrm{O}$ manejo das aves foi idêntico àquele empregado pela indústria avícola. $O$ experimento foi delineado de forma inteiramente casualizada, com dois tratamentos e seis repetições por tratamento. Os tratamentos foram: boxes sem poleiros (T1) e boxes com poleiros (T2).

Poleiros: Foram utilizados 18 poleiros horizontais, três por box, confeccionados com barras principais de madeira, medindo $1,20 \mathrm{~m}$ de comprimento e $3 \mathrm{~cm}$ de espessura. Os poleiros foram fixados a uma altura de $7,5 \mathrm{~cm}$ da cama, através de dois suportes para sustentação. Em cada suporte foi fixado um motivador composto de fios de náilon, com o objetivo de atrair a curiosidade das aves e as motivar a subir nos poleiros. Os poleiros foram instalados radialmente ao centro da área do box, que era a posição da campânula ao início do experimento (FIGURA 1).

Observações Comportamentais: Para a avaliação da Liberdade Comportamental das aves foram observadas as ocorrências de atividades comportamentais incluindo beber, comer, descansar embaixo da campânula, andar, ciscar, deitar e se empoleirar, assim como os eventos de comportamento agonista, através de registro em lista de checagem. As avaliações foram realizadas duas vezes por semana, seis vezes ao dia, sendo três em horários variáveis, com um intervalo de 30 minutos, com variação do horário de início das observações entre 10:00 h e 16:00 h, e três em horários fixos em relação ao pôr do sol, ocorrendo $2 \mathrm{~h}$ e $1 \mathrm{~h}$ antes do sol se pôr e 30 minutos após. As observações foram realizadas através de registro instantâneo do tipo varredura (MARTIN e BATESON, 2002).

Exame Físico: Para a avaliação da Liberdade Sanitária dos frangos foram observadas a condição das penas do peito e a incidência de distúrbios de pernas. Para a quantificação de problemas de empenamento de peito, foram observadas aleatoriamente 10 das aves de cada box aos 40 dias de idade, sendo avaliada a proporção da área do peito sem penas. As aves foram enquadradas nas seguintes classes de condição de empenamento de peito: regular a boa $(0-30 \%$ do peito sem penas), ruim (31-60\%), muito ruim $(61-90 \%)$ e péssima $(91-100 \%)$. As mesmas aves foram avaliadas com relação à presença de distúrbios nas pernas, caracterizadas por desvio de ângulo e/ou aumento de volume nas articulações, soluções de continuidade da pele e/ou anormalidade de movimentação mediante exame. 
Impacto da oferta de poleiros sobre o bem-estar de frangos de corte

FIGURA 1 - VISTA SUPERIOR DA DISPOSIÇÃO DE BEBEDOURO (B), COMEDOURO (C), POLEIROS (P) E MOTIVADORES $(*)$ NOS SEIS BOXES DO TRATAMENTO 2; O CÍRCULO DUPLO REPRESENTA A CAMPÂNULA PRESENTE AO INÍCIO DO EXPERIMENTO; OS BOXES DO TRATAMENTO 1 APRESENTAVAM IGUAL DISTRIBUIÇÃO DE BEBEDOURO, COMEDOURO E CAMPÂNULA. CENTRO DE ESTAÇÕES EXPERIMENTAIS DO CANGÜIRI, UFPR, PINHAIS, PR, 2005.

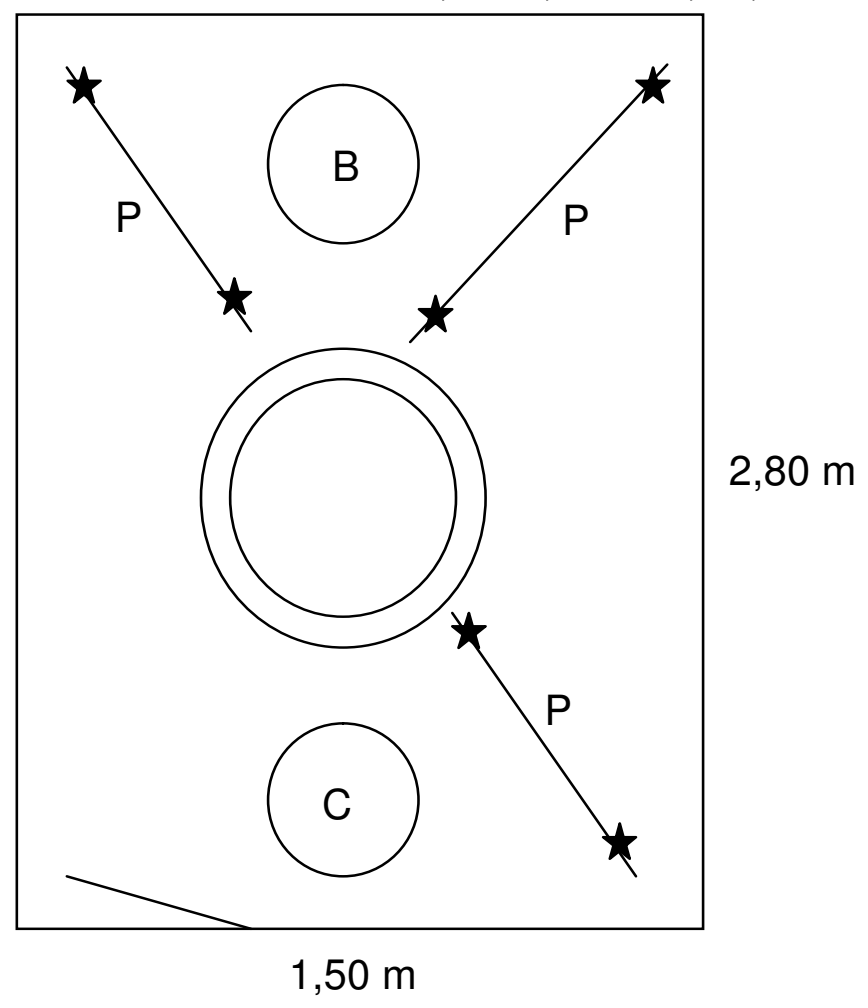

Parâmetros Zootécnicos: Aos 7, 21 e 42 dias de idade, as aves e as sobras de ração foram pesadas e foram avaliadas para o estudo dos seguintes índices zootécnicos: consumo de ração por box, consumo médio individual de alimento, conversão alimentar, ganho de peso médio e peso ao abate.

Análise Estatística: A análise estatística foi realizada através de teste $F$, ANOVA, para os índices zootécnicos e através do teste de MannWhitney para as variáveis comportamentais. Todas as análises foram realizadas com o pacote Statview, (SAS, 1998).

\section{Resultados e discussão}

Os resultados de freqüência de atividades comportamentais entre aves dos diferentes tratamentos são apresentadas na TABELA 1. Observou-se dificuldade das aves em subir no poleiro nas primeiras três semanas de vida, sendo que a altura do poleiro pareceu ser um fator limitante. Houve diferença entre os tratamentos $(P<0,05)$, na quinta e sexta semanas de vida, com relação ao número de ocorrências das atividades de ciscar e comportamento de interação agonista (GRÁFICO 1). Os animais apresentaram maior freqüência de ocorrências de ciscar e menor freqüência de ocorrências de comportamento agonista na presença de poleiros. 

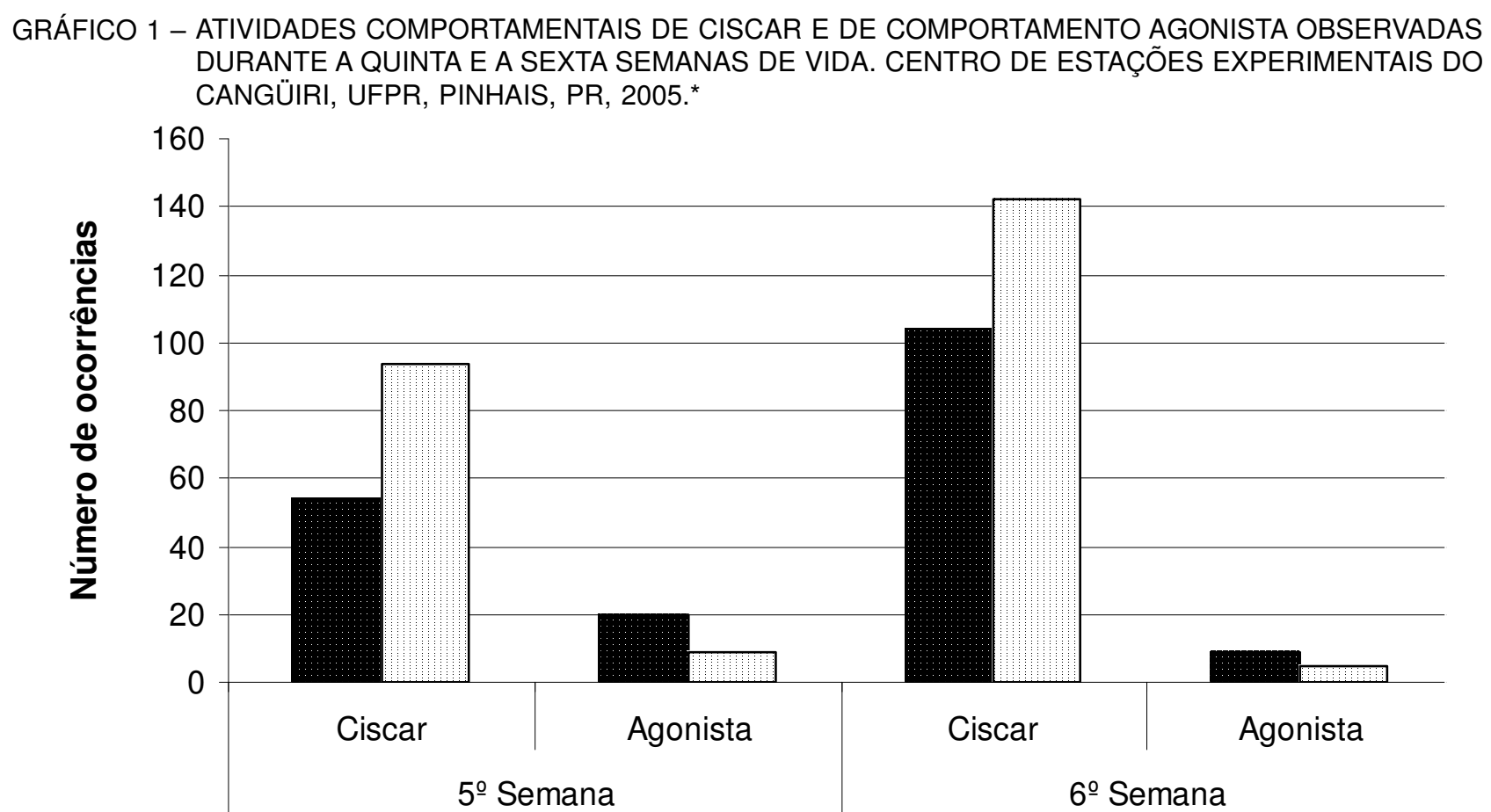

Atividades Comportamentais/Semanas

Sem poleiros $\square$ Com poleiros

Os horários de maior ocorrência de empoleiramento foram 13:00 h (35 ocorrências), 15:00 h (34 ocorrências), 17:00 h (25 ocorrências), 18:00 h (36 ocorrências), posteriormente decrescendo até as 20:20 h (22 ocorrências). Devido ao galpão ser compartilhado com outro experimento, foi mantida iluminação artificial constante, de 24 horas de luz durante toda a fase experimental. Isto provavelmente alterou um potencial ritmo circadiano para o comportamento de se empoleirar dos frangos, para o qual havia uma expectativa de pico nas horas próximas ao pôr do sol. A freqüência de utilização de poleiros aumentou à medida que a idade dos frangos aumentou, sendo que 0 ato de se empoleirar representou $0,3 \%, 2,6 \%$ e $5,1 \%$ de todas as atividades comportamentais observadas na quarta, quinta e sexta semanas de vida, respectivamente. $O$ efeito cumulativo do uso de poleiros pelos frangos, ao longo da vida, foi limitado pela ausência total de empoleiramento nas primeiras três semanas de vida. Entretanto, um período de utilização de poleiros de três semanas significa que algumas aves utilizaram o recurso ambiental durante metade de suas vidas. O número absoluto total de ocorrências de empoleiramento foi de 4, 76 e 111, para a quarta, quinta e sexta semanas, respectivamente. A utilização de poleiros observada foi superior aos valores da freqüência total de empoleiramento relatados por PETTIT-RILEY e ESTEVEZ (2001), de 2,6\%. Estes autores trabalharam avaliando o efeito da densidade animal, utilizando 10 ,
15 e 20 aves $/ \mathrm{m}^{2}$ e o uso de poleiros em diferentes graus angulares de inclinação, verificando o maior uso dos poleiros na terceira e quarta semanas de vida para 0 tratamento com poleiros na horizontal. ESTEVEZ et al. (2002), ao trabalhar com densidade animal de 13,4 aves/ $\mathrm{m}^{2}$, utilizando poleiros resfriados distantes do piso 15 $\mathrm{cm}$ e poleiros em temperatura ambiente a $7,5 \mathrm{~cm}$ e 15 $\mathrm{cm}$ do piso, fizeram comparação entre temperatura e posições nos poleiros e verificaram maior freqüência de empoleiramento na quarta semana, no tratamento com poleiros em temperatura ambiente, distantes do solo $7,5 \mathrm{~cm}$, com menor freqüência de uso nas semanas seguintes. De maneira similar aos resultados obtidos, ESTEVEZ et al. (2002) relataram que, nos tratamentos com poleiros, os frangos demonstraram-se mais calmos e menos agressivos. É possível que ESTEVEZ et al. (2002) tenham observado maior frequência de empoleiramento nas semanas de três e quatro devido à maior densidade de lotação que empregaram $\left(13,4\right.$ aves $\left./ \mathrm{m}^{2}\right)$. Com a utilização da densidade animal de 7,1 aves $/ \mathrm{m}^{2}$ neste experimento, associada a um auge de empoleiramento nas semanas cinco e seis, pode-se sugerir duas hipóteses: (1) que os frangos com menos espaço tenham buscado os poleiros como alívio para suas dificuldades de forma mais precoce e (2) que os frangos com menos espaço tiveram restrições físicas para se empoleirar quando estavam maiores, nas semanas cinco e seis. 
Impacto da oferta de poleiros sobre o bem-estar de frangos de corte

TABELA 1 - NÚMERO DE OCORRÊNCIAS DAS ATIVIDADES COMPORTAMENTAIS NA AUSÊNCIA DE POLEIROS (T1) E NA PRESENÇA DE POLEIROS (T2), AGRUPADAS PARA TRÊS PERÍODOS DE IDADE DOS FRANGOS (ATÉ DUAS SEMANAS, TRÊS A QUATRO SEMANAS E CINCO A SEIS SEMANAS DE VIDA). CENTRO DE ESTAÇÕES EXPERIMENTAIS DO CANGÜIRI, UFPR, PINHAIS, PR, 2005.

\begin{tabular}{|c|c|c|c|c|c|c|}
\hline \multirow{3}{*}{$\begin{array}{c}\text { Atividades } \\
\text { Comportamentais }\end{array}$} & \multicolumn{6}{|c|}{$\begin{array}{c}\text { Ocorrências em seis observaçôes diárias durante quatro dias ao longo de cada } \\
\text { duas semanas de vida das aves }\end{array}$} \\
\hline & \multicolumn{2}{|c|}{ Semanas 1 e 2} & \multicolumn{2}{|c|}{ Semanas $3^{1}$ e 4} & \multicolumn{2}{|c|}{ Semanas 5 e 6} \\
\hline & T1 & $\mathrm{T} 2$ & T1 & T2 & T1 & $\mathrm{T} 2$ \\
\hline Beber & 153 & 166 & 41 & 37 & 239 & 241 \\
\hline Comer & 613 & 609 & 321 & 320 & 727 & 699 \\
\hline Campânula & 1731 & 1771 & 236 & 282 & 0 & 0 \\
\hline Andar & 346 & 389 & 192 & 201 & 715 & 627 \\
\hline Ciscar & 198 & 211 & 79 & 93 & $158^{a 2}$ & $236^{b}$ \\
\hline Deitar & 498 & 244 & 901 & 839 & 2101 & 1954 \\
\hline Empoleirar & 0 & 0 & 0 & 4 & 0 & 177 \\
\hline Agonista & 0 & 0 & 0 & 0 & $29^{a}$ & $14^{b}$ \\
\hline
\end{tabular}

${ }^{1}$ Devido a restrições logísticas, o número de observações realizadas na terceira semana de idade foi $50 \%$ menor do que o programado.

${ }^{2}$ Letras diferentes representam diferença significativa $(P<0,05)$, conforme o teste de Mann-Whitney.

Com a oferta de poleiros observou-se um maior percentual de animais com condição de peito boa, assim como um maior percentual de animais com condição de peito péssima (GRÁFICO 2). Desta forma, os resultados não permitem concluir um efeito nítido dos poleiros sobre a condição das penas do peito. Este achado está de acordo com a literatura (BESSEI, 2006), sendo necessários futuros estudos do efeito de poleiros sobre a condição do peito das aves. Com relação a problemas de pernas, somente uma ave do T1 apresentou deformidade nas pernas. Embora a ave com distúrbio de pernas estivesse alojada em box sem poleiros, o resultado é insuficiente para uma conclusão acerca do efeito da oferta de poleiros sobre a incidência de problemas nas pernas. Como a densidade de lotação utilizada no experimento foi inferior àquela da indústria intensiva, talvez os efeitos negativos do sistema de produção sobre a Liberdade Sanitária das aves tenham sido também inferiores. Este raciocínio parte do conhecimento de que, com o aumento da densidade animal ocorre um aumento na incidência de lesões no peito (FURLAN et al. 2005), sendo que o aumento de problemas de peito indica menor grau de bem-estar animal (PARANHOS DA COSTA, 2003). Desta forma, sugere-se estudos com densidade de lotação similares às empregadas pela indústria com o objetivo de verificar o efeito dos poleiros sobre a Liberdade Sanitária das aves. Deve-se estudar, adicionalmente, se a freqüência de uso dos poleiros observada neste experimento repete-se em maiores densidades de lotação.

A utilização de poleiros tem relação com a base genética dos frangos (EDDIE e KOENE, 2002). Em experimentos utilizando duas linhagens de frangos com bases genéticas diferentes, crescimento rápido ou lento, da primeira à $12^{\circ}$ semana de vida, foi observado que a maior freqüência de empoleiramento foi na terceira, quarta e quinta semanas. De modo geral, os frangos com a genética de crescimento lento utilizaram mais 0 poleiro $(P<0,05)$ que a linhagem de crescimento rápido. Tendo por base a utilização de frangos de crescimento rápido neste experimento, a expectativa era de que os frangos teriam limitações importantes para fazer uso de recursos ambientais exigindo habilidades motoras, tais como poleiros. De fato, BESSEI (2006) relata que recursos como poleiros são pouco utilizados por frangos de corte e não aumentam a atividade das aves. Entretanto, os resultados observados sugerem possibilidades de uso de poleiros e de aumento de atividades como ciscar, mesmo pelas linhagens de crescimento rápido. 
GRÁFICO 2 - CONDIÇÃO DE EMPENAMENTO DO PEITO AO EXAME FÍSICO AOS 40 DIAS DE IDADE. CENTRO DE ESTAÇŐES EXPERIMENTAIS DO CANGÜIRI, UFPR, PINHAIS, PR, 2005.

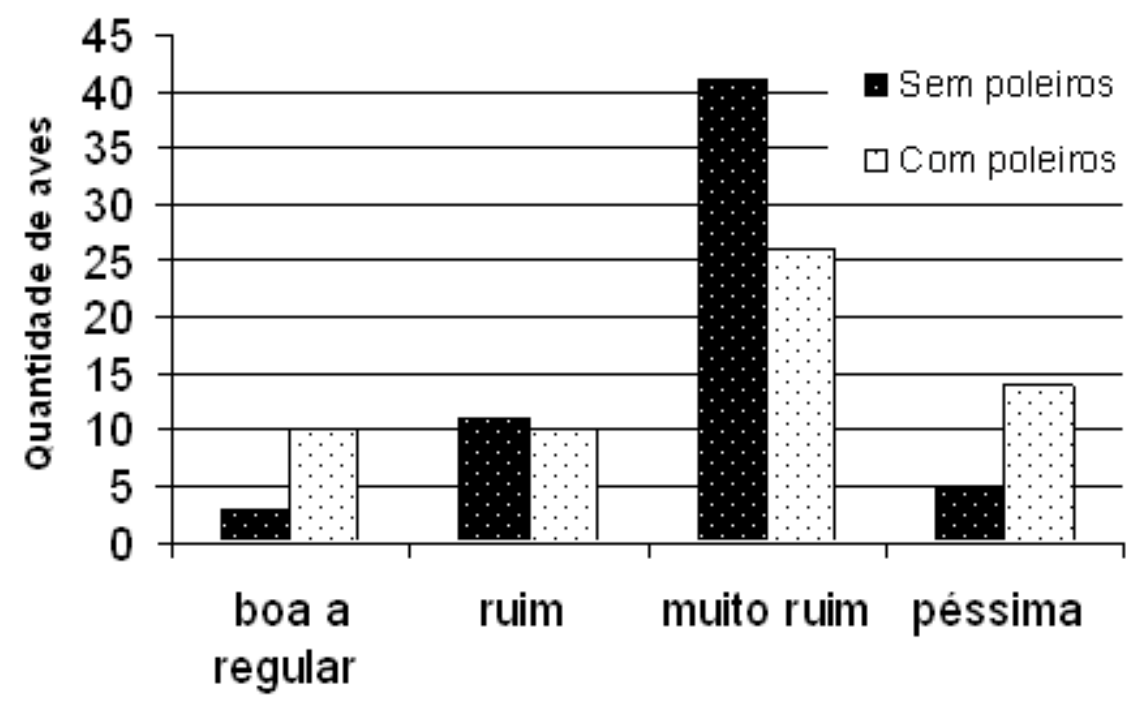

Condição das penas do peito

TABELA 2 - MÉDIA (ERRO PADRÃO) DOS PARÂMETROS ZOOTÉCNICOS DE FRANGOS DE CORTE MANTIDOS EM BOXES DE 4,20 $\mathrm{m}^{2}$ DESDE O PRIMEIRO DIA ATÉ OS 7, 21 E 42 DIAS DE IDADE, CONFORME PESAGEM POR LOTE. CENTRO DE ESTAÇÕES EXPERIMENTAIS DO CANGÜIRI, UFPR, PINHAIS, PR, 2005.

\begin{tabular}{|c|c|c|c|c|c|c|}
\hline \multirow{3}{*}{ Parâmetros zootécnicos } & \multicolumn{6}{|c|}{ Dias de idade } \\
\hline & \multicolumn{2}{|c|}{7} & \multicolumn{2}{|c|}{21} & \multicolumn{2}{|c|}{42} \\
\hline & $\mathrm{T}^{1}{ }^{1}$ & $\mathrm{~T}^{1}$ & T1 & T2 & T1 & T2 \\
\hline $\begin{array}{l}\text { Consumo do lote, } \mathrm{kg} \text { de } \\
\text { racẫoloteiperíodo }\end{array}$ & $\begin{array}{c}3,89 \\
(0,10)\end{array}$ & $\begin{array}{c}4,36 \\
(0,23)\end{array}$ & $\begin{array}{l}37,72 \\
(0,94)\end{array}$ & $\begin{array}{l}37,57 \\
(0,92)\end{array}$ & $\begin{array}{c}148,59 \\
(0,78)\end{array}$ & $\begin{array}{l}147,30 \\
(0,94)\end{array}$ \\
\hline Consumo individual, $\mathrm{g}$ de & 130 & 146 & 1,26 & 1,27 & 5,01 & 4,98 \\
\hline raçấolaveiperíodo & (3) & (7) & $(0,33)$ & $(0,33)$ & $(0,05)$ & $(0,05)$ \\
\hline Conversẫo alimentar, $\mathrm{kg}$ de & 0,88 & 0,98 & 1,59 & 1,57 & 2,23 & 2,33 \\
\hline raçẫolaveiperíodo & $(0,02)$ & $(0,04)$ & $(0,05)$ & $(0,03)$ & $(0,04)$ & $(0,05)$ \\
\hline Peso do lote ${ }^{2}, \mathrm{~kg}$ de peso & 6,10 & 5,82 & 29,49 & 30,0 & 78,65 & 74,57 \\
\hline vivoiloteiperíodo & $(0,06)$ & $(0,31)$ & $(0,33)$ & $(0,20)$ & $(1,37)$ & $(1,65)$ \\
\hline
\end{tabular}

${ }^{1} \mathrm{~T} 1$ = sem poleiros, $\mathrm{T} 2$ = com poleiros; comparação entre $\mathrm{T} 1$ e T2 revelou $\mathrm{P}<0,05$ para conversão alimentar aos sete dias e $\mathrm{P}>0,05$ para os outros parâmetros.

${ }^{2}$ Corrigido para número de aves por lote.

Avaliando-se os resultados dos índices zootécnicos para consumo de ração, ganho de peso, peso ao abate e conversão alimentar aos sete, 21 e 42 dias (TABELA $2)$, percebe-se diferença significativa $(P<0,05)$ para a conversão alimentar aos sete dias, com médias de 0,88 $\pm 0,02$ e 0,98 $\pm 0,015 \mathrm{~kg}$ de ração/ave/período de sete dias, para os tratamentos 1 e 2 respectivamente. A relevância deste achado parece frágil, frente à ausência de diferença significativa entre os tratamentos para todos os demais índices zootécnicos $(P>0,05)$.

\section{Conclusão}

Conclui-se que a oferta de poleiros nas condições deste experimento resulta em melhoria da Liberdade Comportamental no último terço de vida das aves e que o efeito dos poleiros sobre a Liberdade Sanitária deve ser estudado mais detalhadamente. Os resultados sugerem melhoria do grau de bem-estar de frangos de corte devido à oferta de poleiros. Futuras pesquisas devem ser conduzidas no sentido de promover uma 
Impacto da oferta de poleiros sobre o bem-estar de frangos de corte

utilização dos poleiros pelas aves de forma mais precoce, de estudar o impacto dos poleiros sobre a Liberdade Sanitária em situações industriais de maior densidade de lotação e de estudar o comportamento das aves frente aos poleiros em situações de iluminação natural.

\section{Agradecimento}

Registra-se um agradecimento a todos os estagiários do Laboratório de Bem-estar animal - LABEA, UFPR, que auxiliaram durante os períodos de observação das atividades comportamentais das aves.

\section{Referências}

APINCO. Produção de pintos de corte e perspectivas para o ano de 2006. Disponível em: http:// www.avisite.com.br. Acesso em: 12 set. 2006.

BESSEI, W. Welfare of Broilers: a review. World's Poultry Science Journal, v. 62, p. 455-466. 2006.

BROOM, D.M; MOLENTO, C.F.M. Bem-estar animal: conceito e questões relacionadas - revisão. Archives of Veterinary Science, v. 9, n. 2, p. 1-11, 2004.

EDDIE, A.M.B; KOENE, P. Behaviour of fast and slow growing broilers to 12 weeks of age and the physical consequences. Applied Animal Behaviour Science, v. 81, p. 59-72, 2002.

ESTEVEZ I, TABLANTE N, PETTIT-RILEY R.L, CARR L. Use of cool perches by broiler chickens. Poultry Science, v. 81, n. 1, p. 62-69, 2002.

FRASER, A.F.; BROOM, D.M. Farm Animal Behaviour and Welfare. Oxon: CABI Publishing, 2002, p. 437.

FURLAN. R.L; MACARI, M.; PARANHOS DA COSTA, M.J.R. Bem-estar das aves e suas implicações sobre o desenvolvimento e produção - Fórum I Internacional de Avicultura. Foz do Iguaçu, 17 a 19 - Agosto de 2005.

JENSEN, P. The Ethology of Domestic Animals - An Introductory Text, Oxon: CABI Publishing, 2002, p. 218.

LEI 14.037, 2003 - Constituição do Estado do Parana Código Estadual de Proteção aos Animais. Disponível em:http://www.ceaam.net/lef/pr/LegisEstadual.htm. Acesso em: 13 de Set de 2006.
LEI 11.977, 2005 - Código de Proteção aos Animais do Estado de Sao Paulo. Disponível em:http:// www.propg.ufscar.br/pdf/etica_animais/lei11977.pdf. Acesso em: 13 de Set de 2006.

MARTIN, P; BATESON, P. Measuring behaviour - an introductory guide. $2^{a}$ Edição, Cambridge University Press, Cambridge, Reino Unido. 2002. 222 p.

MITCHELL, M.A.; KETTLEWELL, P.J. Sistemas de transporte e bem-estar de frangos de Corte. In: CONFERÊNCIA APINCO DE CIÊNCIA E TECNOLOGIA AVÍCOLAS, 2003. p.189 - 197.

MOLENTO, C.F.M. Bem-Estar e Produção Animal: Aspectos Econômicos - Revisão. Archives of Veterinary Science, v. 10, n. 1, p. 1-11, 2005.

MOLENTO, C.F.M. Medicina Veterinária e Bem-Estar Animal. Revista CFMV. Brasília: Conselho Federal de Medicina Veterinária, v.28/29, p.15 - 20, 2003.

MOLENTO, C.F.M. Rethinking the Five Freedoms. In: । CONGRESSO INTERNACIONALDE CONCEITOS EM BEMESTARANIMAL. 2006, Rio de Janeiro, Anais... Rio de Janeiro: Sociedade Mundial de Proteção Animal, 2006, CD.

NEWBERRY, R.C. Environmental enrichment: increasing the biological relevance of captive environments. Applied Animal Behaviour Science. V. 44, p. 229-243, 1995.

PARANHOS DA COSTA, M.J.R. Princípios de Etologia aplicada ao bem-estar das aves. In: CONFERÊNCIA APINCO DE CIÊNCIA E TECNOLOGIA AVÍCOLAS, 2003.

PETTIT-RILEY, R.L., ESTEVEZ I. Effects of density on perching behavior of broiler chickens. Applied Animal Behaviour Science. V. 71, p.127-140, 2001.

SAS. Statview for Windows, SAS Institute Inc., Version 5.0.1, 1998.

SHEPPARD, A.; EDGE, S. Economic and Operational Impacts of the Proposed EU Directive laying down Minimum Standards for the Protection of Chickens kept for Meat Production. Research undertaken for Defra by the University of Exeter Centre for Rural Research and ADAS, 2005. Disponível em: www.exeter.ac.nk/err. Acesso em: 13 de Ago de 2006.

STATUTORY INSTRUMENT No. 1870, 2000. Prevention of Cruelty, England - The Welfare of Farmed Animals (England). Regulations, 2000. Great Britain. Disponível em: http://www.defra.gov.uk/animalh/welfare/farmed/ index.htm e http://www.opsi.gov.uk/si/si2000/ 20001870.htm. Acesso em: 11 de Ago de 2006.

Recebido para publicação:

$20 / 09 / 2006$

Aprovado: 